\title{
Determinación de las propiedades físicas y mecánicas de dos muestras de palma chonta en el municipio de Tadó, Chocó, Colombia
}

\section{Determination of the physical and mechanical properties of two samples of palm chonta in the municipality of Tadó, Chocó, Colombia}

\section{Jovanny Mosquera-Pino*}

\section{Resumen}

En este estudio se da a conocer los resultados obtenidos en los ensayos de las propiedades físicas y mecánicas de segmentos del estipe de palma memé Wettinia quinaria (m1) y palma barrigona Iriartea deltoidea (m2) en el año 2015 en el municipio de Tadó, Chocó, Colombia. Los ensayos físicos y mecánicos se llevaron a cabo con base en la metodología estipulada en la Norma América ASTMD 143-2000, método de prueba a flexión para probetas de pequeñas dimensiones y las Normas Técnicas Colombianas (NTC), siendo estos contenidos de humedad, contracción y densidad para el ensayo físico y flexión estática y comprensión paralela para el ensayo mecánico. Los resultados mostraron que la densidad fue de $1.34 \mathrm{~g} / \mathrm{cm}^{3}$ para la muestra uno (m1) y $1.36 \mathrm{~g} / \mathrm{cm}^{3}$ para la muestra dos (m2), siendo las dos palmas maderas clasificadas como muy pesadas. El módulo de roptura fue de $1640,3 \mathrm{~kg} / \mathrm{cm}^{2}$ y $1547,22 \mathrm{~kg} / \mathrm{cm}^{2}$ lo que las clasifica como flexión alto, teniendo ambas muestras una dureza muy baja por debajo de $200 \mathrm{~kg}$ de comprensión de los lados. El presente estudio pretende aportar elementos necesarios para conocer el comportamiento físico y los niveles de resistencia en la madera de palma memé y palma barrigona, como una contribución al conocimiento tecnológico de las especies de palmas utilizadas de manera tradicional por las comunidades negras del Alto San Juan

Palabras clave: Chonta, Densidad, Dureza, Madera, Palma, Propiedades físicas, Propiedades mecánicas.

\begin{abstract}
This study we present the results obtained in the trials of the physical and mechanical properties of the palma mete Wettinia quinaria (m1) and palma barrigona Iriartea deltoidea (m2) stipe segments in the year 2015 in the municipality of Tadó, Chocó.
\end{abstract}

Investigador Asociado II, Instituto de Investigaciones Ambientales del Pacífico (IIAP), Quibdó, Chocó, Colombia. e-mail: jmosquera@iiap.org.co 


\section{Bioetnia Volumen 13, 2016}

The physical and mechanical tests were carried out based on the methodology stipulated in the American Standard ASTM D 143-2000. Bending test method for small specimens and the Colombian Technical Standards NTC, being these contents of humidity, contraction and density for the physical test and static flexion and parallel understanding for the mechanical test. The results showed that the density was $1.34 \mathrm{~g} / \mathrm{cm}^{3}$ for the sample one (m1) and $1.36 \mathrm{~g} / \mathrm{cm}^{3}$ for the sample two (m2), the two palms being classified as very heavy. The ropture modulus was $1640.3 \mathrm{~kg} / \mathrm{cm}^{2}$ and 1547.22 $\mathrm{kg} / \mathrm{cm}^{2}$ which classifies them as high bending, both samples having a very low hardness below $200 \mathrm{~kg}$ of understanding of the sides. The present study intends to provide the necessaryelements to know the physical behavior and resistance levels in the Palma Meme and Palma Barrigona wood, as a contribution to the technological knowledge of the palm species traditionally used by the black communities of Alto San Juan.

Keywords: Chonta, Density, Hardness, Mechanical properties, Palm, Physical properties, Wood.

\section{Introducción}

Dentro de las propiedades tecnológicas de las maderas, las físico-mecánicas son de las más importantes, porque se utilizan como índices para evaluar si las características o calidad de una madera son adecuadas para un uso particular (Panshin y De Zeew 1980, Brazier y Howell 1979, Schniewind 1989). La determinación de las propiedades mecánicas es un proceso que requiere de equipo, personal calificado, un programa de ensayos, material y tiempo para realizar los ensayos destructivos. Los estudios anatómicos de la madera pueden significar un ahorro de tiempo y dinero y, por la relación que tienen con las propiedades mecánicas, permiten predecirlas.

En otro matiz, cuando se procesa una madera "nueva" para la industria forestal y se intenta comercializarla, la información publicada es limitada sobre sus características o estudios tecnológicos. La correlación estructura-propiedad determinada con un número reducido de especímenes, puede ser aplicada para proponer índices de las propiedades físico-mecánicas de cualquier madera (Bárcenas-Pazos et al. 2005).

El valor de la densidad básica se ha aplicado para estimar algunos de valores de resistencia mecánica, como módulos de elasticidad y de ruptura en flexión y el esfuerzo al límite proporcional en compresión perpendicular, altamente relacionados con la densidad básica (Brown et al. 1952, Williamson 1975, Panshin y De Zeeuw 1980). La relación de la densidad básica con diferentes propiedades físicas y mecánicas de la madera ha sido estudiada desde principios del siglo pasado.

La madera se podría idealizar como un conjunto de elementos celulares de sección circular orientados longitudinalmente y seccionados por los radios dispuestos a manera de pequeñas cuñas que parten del centro hacia el perímetro. Dentro de los elementos celulares longitudinales, las fibras son los más robustos y a ellas se debe gran parte de la resistencia de la madera (Panshin y De Zeew 1980, Guerrero et al. 1992). Las fibras funcionan como columnas individuales, las cuales reciben parte de las acciones y se apoyan en las células vecinas, porque las paredes adyacentes están cementadas (Bodig y Jane 1982, Panshin y De Zeew 1980).

En el caso de la madera, esta ha sido un material indispensable en el desarrollo de la industria nacional fundamentalmente en la construcción, debido a que posee características que la hacen ventajosa en comparación con otros materiales comúnmente utilizados como el acero y el hormigón. Sin embargo, su utilización en el país se ha reducido, principalmente por el desconocimiento de sus excelentes características y por una pérdida de credibilidad en el material que ha tenido su origen en el mal manejo de desventajas naturales 
como son su biodegradabilidad y su combustibilidad (Klinger et al. 2002).

El presente estudio pretende aportar elementos necesarios para conocer el comportamiento físico y los niveles de resistencia en la madera de palma memé Wettinia quinaria y palma barrigona Iriartea deltoidea, como una contribución al conocimiento tecnológico de las especies de palmas utilizadas de manera tradicional por las comunidades negras del Alto San Juan con lo cual se podrá sugerir, probar, validar y difundir las posibilidades de diversificación de los productos forestales de la región lo que repercutirá en un beneficio directo a los poseedores y transformadores del recurso.

\section{Materiales y método}

Área de estudio. El material vegetal procedió de dos especies de palmas memé y barrigona perteneciente a la familia Aracaceae, los cuales se seleccionaron en los bosques colectivos del Consejo Comunitario Mayor del Alto San Juan (ASOCASAN) en la comunidad de Playa de Oro, ubicada en el municipio de Tadó, Chocó, Colombia. El municipio de Tadó está ubicado en la parte oriental del departamento del Chocó, dentro de la zona del Alto San Juan (Figura 1). La cabecera municipal de Tadó se encuentra a 66 km de Quibdó la capital del departamento del Chocó y a 549 km de la ciudad de Bogotá. Tiene

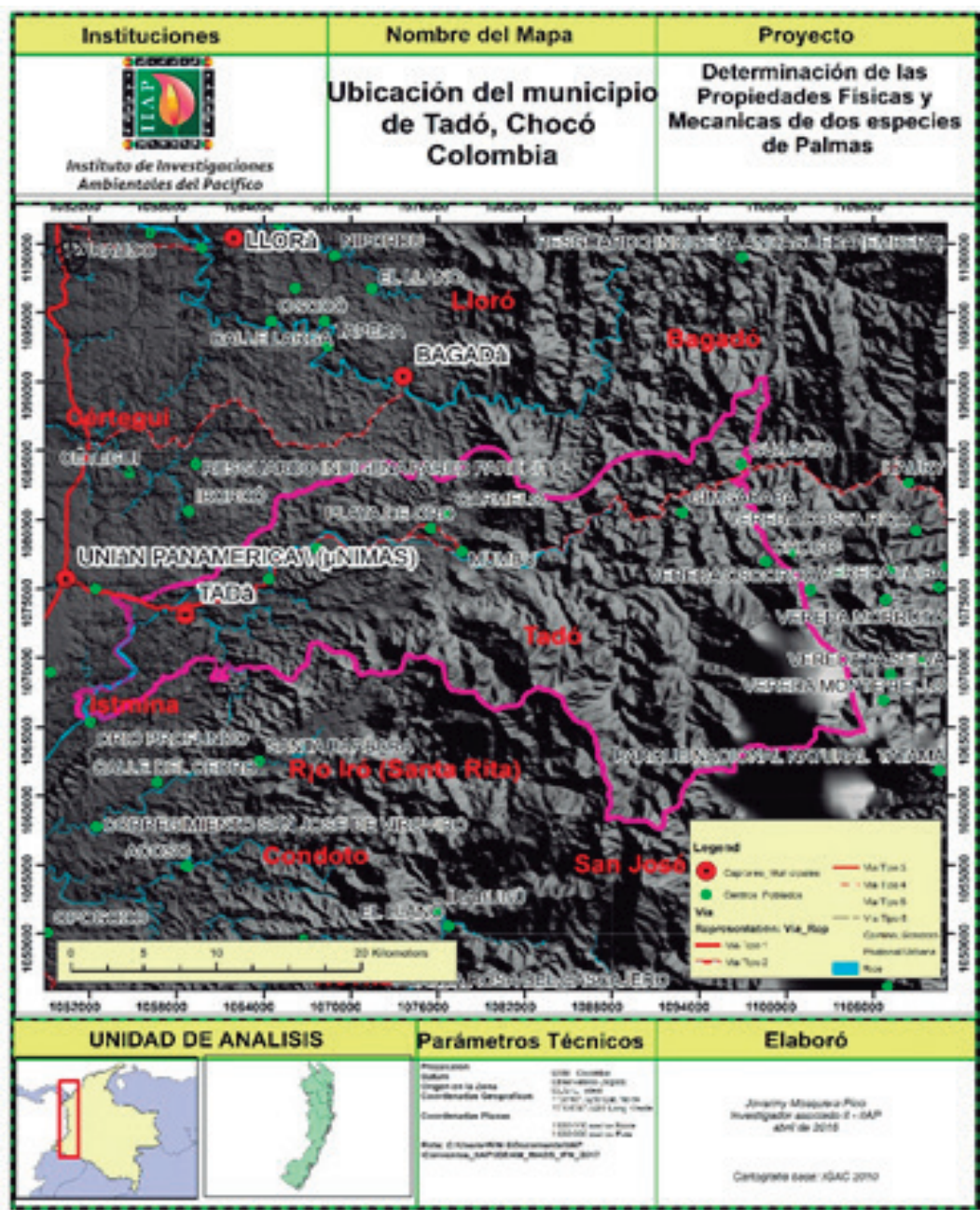

Figura 1. Ubicación del municipio de Tadó. 
un área aproximada de $878 \mathrm{~km}^{2}$. Geográficamente se ubica a $\operatorname{los} 05^{\circ} 15^{\prime} 52,9^{\circ}$ de latitud norte y a $076^{\circ} 33^{\prime} 37.7^{\prime}$ ' de longitud oeste, con una altura de $90 \mathrm{msnm}$ y posee una temperatura media de $28^{\circ} \mathrm{C}$ (Alcaldía Municipal de Tadó, 2005).

Ramírez y Galeano (2012) en su estudio sobre comunidades de palmas en dos bosques del Chocó, citan que la estructura de la población encontrada tanto en Tutunendo como en Angostura, presentan bajo porcentaje de plántulas con respecto a los juveniles y adultos, lo cual podría sugerir que existen problemas de reclutamiento a nivel general en la comunidad, resultado que reportan los moradores de la zona citando que a finales de la década de 1980, se inició una fuerte presión por cosechar chonta debido a la gran demanda comercial en los departamentos de Risaralda y Valle del Cauca.

Por su ubicación geográfica, la zona representa interés ecosistémico, geomorfométricos, geoestructurales y climatológicos; así se define el corredor del Alto San Juan como el área de influencia de la parte alta de la cuenca (Tadó, Santa Cecilia-Pueblo Rico) incluyendo las partes altas de las subcuencas de los ríos Iró, Condoto y Tamaná. Los bosques aún persisten en esta zona pese a la intensidad de la actividad minera $\mathrm{y}$ forestal.

Metodología. Los ensayos físicos y mecánicos se realizaron en la Facultad de Ciencias Agropecuarias del Laboratorio de Productos Forestales de la Universidad Nacional de Colombia con sede en la ciudad de Medellín, apoyado en la metodología estipulada en la Norma América ASTM D 1432000, método de prueba a flexión para probetas de pequeñas dimensiones y las Normas Técnicas Colombianas (NTC).

\section{Equipos utilizados:}

- Balanza digital marca PTT A2000 con capacidad de pesar con precisión de 0,01 gramos.

- Horno de secado marca 1Dies con ventilación forzada con precisión de $\pm 1^{\circ} \mathrm{C}$.

- Micrómetros marca TESA con precisión para medir de $0,01 \mathrm{~cm}$.

- Maquinaria para el aserrado, dimensionado y maquinado de madera.

- Máquina Universal para ensayos mecánicos de madera a escala de pobretería Tinius Olsen.

Selección de especies de palmas chonta. Para la colecta del material se siguió las recomendaciones de la Norma COPANT 458 (Comisión Panamericana de Normas Técnicas) referida a la selección y colección de muestras destinadas al estudio de las propiedades físicas y mecánicas de la madera. La selección de los estípites se realizó partiendo del hecho que las variaciones entre las palmas no son significativas en relación con la variación dentro de la misma especie, por tanto, se tomaron más muestra de cada palma y menos muestras de diferentes individuos (Figura 2).
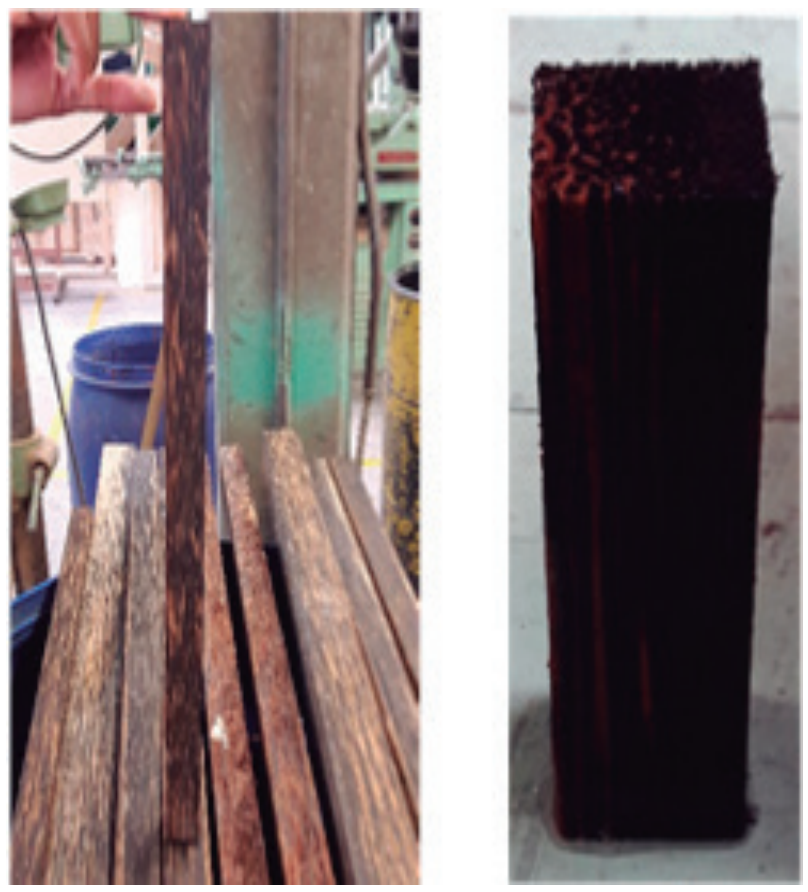

Figura 2. Muestras y probetas del estípite.

Cálculo de propiedades físicas. De la muestra de madera, se extrajeron nueve probetas por muestra de la parte exterior y más cinco de la parte central, las cuales fueron pesadas en una balanza digital con precisión de una centésima de gramo, se midieron sus dimensiones tanto ancho, espesor y largo con micrómetro y después se determinó su 


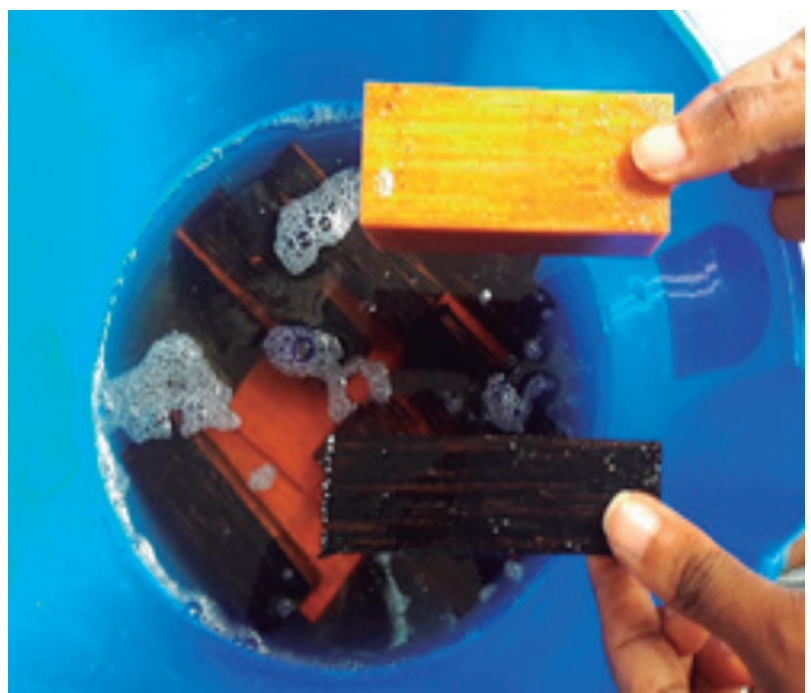

Figura 3. Determinación del contenido de humedad.

volumen para luego ser llevadas a un horno con precisión de $\pm 1^{\circ} \mathrm{C}$ y a una temperatura de $103 \pm 2^{\circ}$ durante 24 horas; las muestras fueron pesadas y nuevamente introducidas al horno durante 20 horas más hasta que el peso fue constante. Del procedimiento anterior se extrajeron los datos de contenido de humedad, contracción y densidad.

Contenido de humedad. Para el presente estudio se hizo el ajuste a un contenido de humedad del 12\%, como lo recomiendan las normas internacionales (Figura 3).

Cálculo de propiedades mecánicas. Para evaluar las propiedades mecánicas se llevó la madera a un contenido de humedad seca al aire

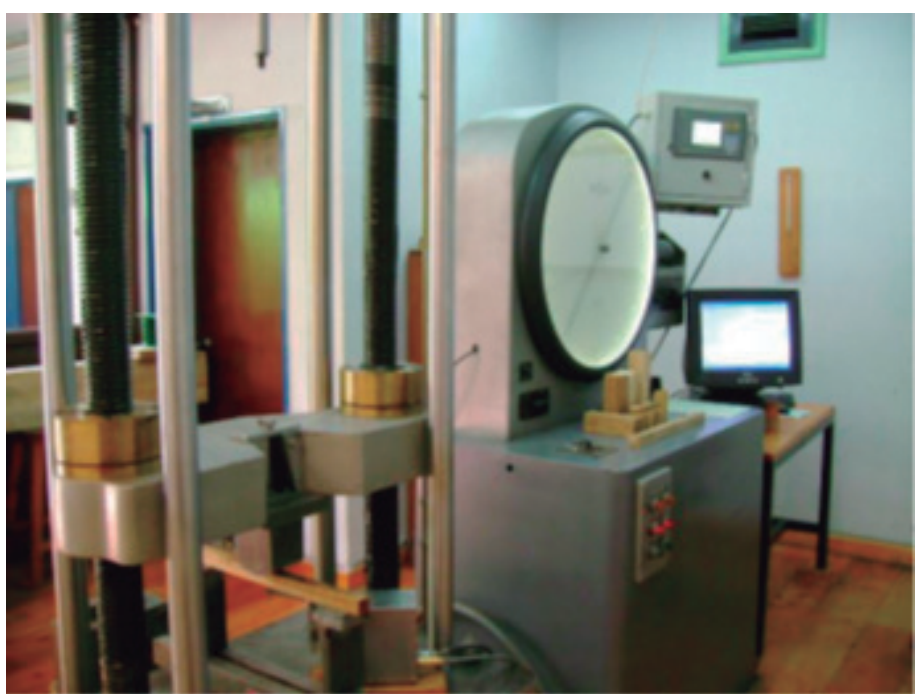

Figura 4. Máquina universal.

(cercano al 12\%), se prepararon probetas de las dimensiones establecidas por las normas para evaluar cada propiedad, y las probetas se hicieron teniendo en cuenta que debían estar libres de defectos tales como nudos, médula, fisuras, aristas faltantes (Figura 4).

Lo ensayos mecánicos se realizaron en una máquina Universal para ensayos mecánicos de madera a escala de pobretería Tinius Olsen con una capacidad de carga de $15.000 \mathrm{~kg}$ y la cual permite obtener directamente gráfico de una curva carga vs. deformación. Así entonces, la caracterización de las propiedades mecánicas de la madera se llevó a cabo teniendo en cuenta la clasificación propuesta por las normas ASTM (Figura 5).

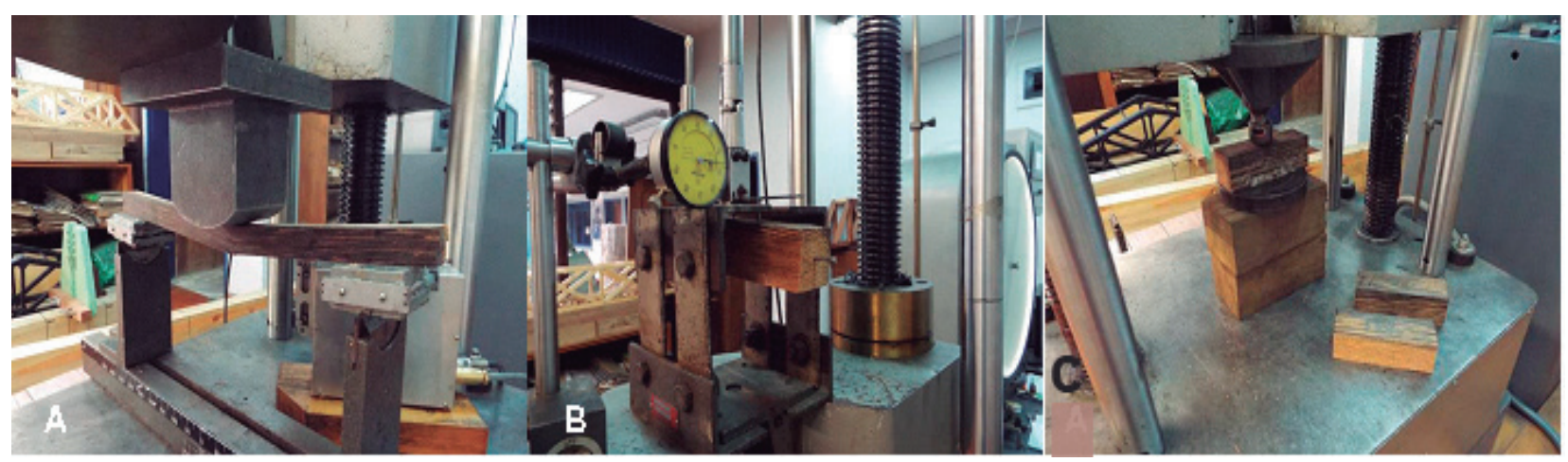

Figura 5. A. Ensayo de flexión estática. B. Ensayo comprensión perpendicular. C. Ensayo de comprensión paralela a las fibras. 


\section{Bioetnia Volumen 13, 2016}

$$
\mathrm{CH} \%=\frac{100(\mathrm{~A}-\mathrm{B})}{\mathrm{B}}
$$

En donde:

$A=$ masa original en gramos

$B=$ masa seca en horno en gramos

$E L P=\frac{1.5^{*} \mathrm{~L} * \mathrm{CLP}}{\mathrm{b}^{*} \mathrm{~h} 2}\left(\mathrm{~kg} / \mathrm{cm}^{2}\right)$

$M O R=\frac{1,5^{*} \mathrm{~L} * \mathrm{~cm}}{\mathrm{~b}^{*} \mathrm{~h} 2}\left(\mathrm{~kg} / \mathrm{cm}^{2}\right)$

$M O E=\frac{0,25 \mathrm{~L} 3 * \mathrm{CLP}}{\mathrm{d}^{*} \mathrm{~b}^{*} \mathrm{~h} 3}\left(\mathrm{~kg} / \mathrm{cm}^{2}\right)$

Dónde:

$E L P$ : Esfuerzo al límite proporcional

MOR: Módulo de ruptura

MOE: Módulo de elasticidad

CLP: Carga al límite proporcional

$C M$ : Carga máxima

$b$ : Distancia tomada entre caras radiales $(\mathrm{cm})$

$h$ : Espesor de la probeta $(\mathrm{cm})$

$d$ : Deformación

L: Luz entre apoyos

Tratamiento estadístico y análisis de la información. Los datos obtenidos de las pruebas físicas se integraron con respecto a la media y coeficiente de variación, mientras que con los resultados de los ensayos mecánicos para identificar diferencias entre los estados de humedad (verde y seco), se realizaron pruebas de t así como análisis de varianza y pruebas de Tukey para la resistencia unitaria máxima por plano (radial y tangencial) y estado de humedad de la madera (verde y seco).

Los datos obtenidos en los diferentes ensayos fueron procesados mediante el programa Microsoft Excel, realizando los cálculos necesarios para la obtención de los módulos y esfuerzos, según lo dictan las normas antes mencionadas. Para la determinación de usos potenciales se realizó siguiendo la metodología propuesta por Klinger y Talero (2001), la cual se fundamenta en las propiedades físicas y mecánicas de cada madera. Luego se realizó la consulta de las diferentes propiedades mecánicas en la base de datos, utilizando los rangos de consulta propuestos por Cifuentes et al. (2005). Con base en estos listados de especies con propiedades mecánicas similares se evaluaron los usos correspondientes, para asignar los de la especie en estudio. La idoneidad estadística de la muestra utilizada para cada ensayo se evaluó confrontando los coeficientes de variación calculados con los máximos valores admisibles según la ASTM.

\section{Resultados y discusión}

Resultados de la prueba física. Acontinuación se presentan los diferentes parámetros utilizados para caracterizar la condición física del estipe de palma utilizando los valores promedio (Tabla 1).

Los resultados obtenidos para la densidad básica de las dos muestras extraídas del tronco de las palmas revelan que no hay diferencias significativas y que su densidad es $0,79 \mathrm{~g} / \mathrm{cm}^{3}$ y para muestras extraídas del centro del fuste su densidad fue $0,13 \mathrm{~g} / \mathrm{cm}^{3}$ (muestras 11-15) con contenidos de humedad hasta de $600 \%$ y $30 \%$ aproximadamente para la zona exterior del fuste. Sin embargo, la densidad al $12 \%$ de humedad fue 1,34 y 1,36 y se puede clasificar para las dos muestras como madera muy pesada (Tabla 2).

En relación con la contracción, esta varía más dentro del mismo fuste que entre las muestras, porque hacia el córtex blando su contracción es elevada (30\%), mientras que hacia la epidermis endurecida es del orden del $12 \%$.

Por otra parte, se puede decir que el estípite de las dos especies de palma es de alta durabilidad natural, maderas con densidades entre $0.8 \mathrm{~g} / \mathrm{cm}^{3} \mathrm{y}$ $1.12 \mathrm{~g} / \mathrm{cm}^{3}$. Alta durabilidad natural (excepto la 
Palma chonta y propiedades física y mecánicas. J Mosquera-Pino

Tabla 1. Resultados de la prueba física

\begin{tabular}{lrrrrrr}
\hline Muestra & $\begin{array}{c}\text { Contenido de } \\
\text { humedad (\%) }\end{array}$ & \multicolumn{4}{c}{ Densidad $\left(\mathbf{g} / \mathbf{c m}^{3}\right)$} & \multirow{2}{*}{$\begin{array}{c}\text { Contracción } \\
\text { valor total (\%) }\end{array}$} \\
\cline { 3 - 6 } & en verde & seca al aire & básica & seca al horno & valor \\
\hline Media & 183,614 & 1,269 & 1,029 & 0,797 & 0,9 & 12,68 \\
\hline Desviación estándar & 287,197 & 0,131 & 0,447 & 0,364 & 0,402 & 5,1 \\
q & 98,125 & 0,042 & 0,152 & 0,119 & 0,131 & 1,666 \\
cv & 1,564 & 0,103 & 0,434 & 0,457 & 0,449 & 0,402 \\
\hline
\end{tabular}

Tabla 2. Densidad básica de las muestras

\begin{tabular}{|c|c|c|c|}
\hline \multicolumn{4}{|c|}{ Densidad ( $12 \%$ de contenido de humedad) } \\
\hline \multirow[t]{2}{*}{ Clasificación } & \multirow{2}{*}{$\begin{array}{l}\text { Densidad } \\
\left(\mathrm{g} / \mathrm{cm}^{3}\right)\end{array}$} & \multicolumn{2}{|c|}{ Muestra } \\
\hline & & 1 (memé) & 2 (barrigona) \\
\hline Muy liviana & $<0,35$ & & \\
\hline Liviana & $0,36-0,55$ & & \\
\hline $\begin{array}{l}\text { Medianament } \\
\text { pesada }\end{array}$ & $0,56-0,75$ & & \\
\hline Pesada & $0,76-1$ & & \\
\hline Muy pesada & $>1$ & 1,34 & 1,36 \\
\hline
\end{tabular}

albura), no requiere tratamiento preservativo. Generalmente se usan como pisos (entablados, parquetería, machihembrado, etc.), pasos de escaleras, elementos torneados (barandas, pasamanos) y en forma de láminas como chapas (Junta del Acuerdo de Cartagena 1984). Por ser una madera de alta durabilidad y usada en construcción no estructural y cuyo uso final puede ser madera vista o cubierta por pintura u otro material, madera para piso, resistente al desgaste y baja contracción.

La densidad básica es el indicador más usado para determinar el rendimiento y la calidad de la madera (Dickson y Walter1997); se considera la característica que mayor información aporta sobre la calidad de la madera, porque existe una buena relación entre ella y la mayoría de las características físico-mecánicas (Gutiérrez y Plaza, 1967). Por otro lado, la densidad es una propiedad que muestra una amplia variación entre y dentro de las especies (Zobel y Van Buijtenen 1989), es resultado del tamaño de los vasos y de la cantidad de estos, del espesor de la pared celular, del diámetro de las fibras y de la composición química de la madera, de tal forma que dos maderas con densidades similares pueden ser muy distintas en cuanto a las características anteriores.

La densidad de la madera es un aspecto importante a considerar durante el proceso de combustión (Coronel 1994). El uso de maderas con densidades bajas para la producción directa de energía en forma de calor implica una quema rápida y una menor producción de energía por unidad de volumen, a diferencia de maderas con densidades altas. Sin embargo, densidades muy altas implican dificultad para iniciar la combustión del material (Teixeira et al. 2002). Según Cunha et al. (1989) no hay una correlación entre densidad básica y el poder calorífico. Sin embargo, en relación con el volumen de madera que se quemará, la densidad se relaciona positivamente con el contenido calórico de la madera.

Resultados de la prueba mecánica. Todos los ensayos tuvieron una variación mayor entre las probetas extraídas del córtex blando de la zona externa del tronco, mientras que no fueron notorias las diferencias entre muestras. Incluso en algunos ensayos no fue posible la obtención de datos, ya que cuando se le aplicaba carga, la probeta se deformaba sin soportar dicha carga, como fue el caso de la compresión perpendicular y la cizalladura.

En relación con la flexión estática, el valor analizado fue el módulo de ruptura (MOR), $\mathrm{kg} /$ $\mathrm{cm}^{2}$. La propiedad de flexión estática se refiere a la 


\section{Bioetnia Volumen 13, 2016}

resistencia que ofrece la madera a cargas, cuando es utilizada como una viga (Torelli 1982). En la flexión estática fue el ensayo donde se registraron los mejores valores, logrando resultados que clasificaría el material de acuerdo con las normas ASTM como clase de resistencia alta (1260-1520) (Tabla 3), siendo un esfuerzo admisible que lleva al módulo de roptura que tiene una variabilidad natural resultante de las condiciones climáticas locales y las características de las especies.

En relación con la comprensión paralela, la muestra número dos (barrigona) arrojó un valor de $146,83\left(\mathrm{~kg} / \mathrm{cm}^{2}\right)$, valor superior a la muestra número uno (memé) que obtuvo 124,84 (kg/ $\left.\mathrm{cm}^{2}\right)$,

Tabla 3. Flexión (Módulo de roptura)

\begin{tabular}{lc}
\hline \multicolumn{2}{c}{$\begin{array}{c}\text { Flexión (Módulo de roptura al 12\% del } \\
\text { contenido de humedad) }\end{array}$} \\
$\begin{array}{l}\text { Clasificación } \\
\text { Flexión }\left(\mathrm{kg} / \mathrm{cm}^{2}\right)\end{array} \frac{\text { Muestra }}{\mathbf{1} \text { (memé) } \mathbf{2} \text { (barrigona) }}$ \\
\hline Muy bajo & $<400$ \\
\hline Bajo & $401-800$ \\
\hline Mediano & $801-1350$ \\
\hline Alto & $1351-1800$ \\
\hline Muy alto & $>1800$ \\
\hline
\end{tabular}

siendo bajo el esfuerzo límite proporcional, lo cual se puede explicar en las diferencias en condiciones de crecimiento de las especies, condiciones de sitio y una posible mezcla de madera de albura y duramen en las probetas que potencialmente influyen en los resultados.

\section{Conclusión}

Para realizar un trabajo que permita resultados más consecuentes con el uso del estipe de palma, se recomendaría separar la zona del centro y solo efectuar los ensayos a la parte exterior del tronco aunque implicara realizar los ensayos por fuera de la norma y crear un procedimiento estandarizado para este tipo de material, porque como se observa en todos los resultados, la influencia de la parte blanda sobre la densidad y las propiedades mecánicas que pudiera tener la zona exterior, está siendo fuertemente afectada.

Debido a que las probetas deben tener un ancho y espesor de $5 \mathrm{~cm}$ para ensayo de propiedades mecánicas de la madera, fue imposible obtener probetas solo con la parte dura del tronco, lo que afectó considerablemente los resultados. Se ha encontrado que las maderas livianas por ser más porosas contienen una mayor cantidad de agua que las maderas pesadas y la albura, por estar conformada por células cuya función es la conducción de agua, presenta un contenido de humedad mayor que el duramen. La albura puede aumentar más del $100 \%$ de su masa en agua (Klinger et al. 2002).

De acuerdo con los resultados del laboratorio, memé y barrigona presentan densidad y módulo de roptura por encima de abarco, algarrobo, carrá y chanul, las cuales se categorizan como madera moderadamente pesada, siendo especies de resistencia mecánica de mediana a alta. Contrario a los resultados de dureza y comprensión paralela, los cuales se encuentran por debajo de las especies antes mencionadas categorizándolas como muy bajo.

La chonta es de secado rápido, de alta calidad, sin sufrir contracciones o pandeos. Dura 35 días para llegar a un equilibrio con el ambiente, tiene mayor pérdida de humedad en los primeros 14 días. "Se considera una madera extremadamente pesada y tiene buena resistencia a flexión, compresión y dureza extrema. Requiere de sierras especiales para trabajar. La madera es rica en taninos que parecen tener un efecto contra microbios" (WWF 2013).

\section{Literatura citada}

Alcaldía Municipal de Tadó. 2005. Esquema de Ordenamiento Territorial, Tadó, Chocó.

IIAP, PNUMA, Natural Justice. 2012. Protocolo comunitario biocultural para el territorio del Consejo Comunitario 


\section{Palma chonta y propiedades física y mecánicas. J Mosquera-Pino}

Mayor del Alto San Juan (ASOCASAN). Tadó, Chocó, Colombia. Quibdó: IIAP, PNUMA, Natural Justice. URL disponible en: http://www.pnuma.org/publicaciones/ PCB\%20ASOCASAN espanol 2012.pdf

Bárcenas-Pazos, GM, Ortega-Escalona F, Ángeles-Álvarez G, Ronzón-Pérez P. 2005. Relación estructura-propiedades de la madera de angiospermas mexicanas. Ecosistemas y Recursos Agropecuarios. 21 (42): 45-55. URL disponible en: http://www.redalyc.org/pdf/3586/Resumenes/ Resumen 358636309001 1.pdf

Brazier JD, Howell RS. 1979. The use of a breast-height core for estimating selected whole-tree properties of Sitka spruce. Forestry. 52 (2): 177-85. URL disponible en: https://academic.oup.com/forestry/article-abstrac$\mathrm{t} / 52 / 2 / 177 / 526382$ ? redirectedFrom $=$ fulltext

Bodig J, Jayne BA. 1982. Mechanics of wood and wood composites. New York: Van Nostrand Reinhold Co. Inc.; $712 \mathrm{pp}$.

Brown HP, Panshin AJ, Forsaith CC. 1952. Textbook of wood technology. Volume II. The physical, mechanical, and chemical properties of the commercial woods of the United States. New York: McGraw-Hill Book Company, Inc.; $783 \mathrm{pp}$.

Cifuentes FJH, Medina N, Polanco C. 2005. Estudio de las propiedades físicas y mecánicas de Eucalyptus tereticornis y Tectona grandis, procedencia Monterrubio, Magdalena. Tesis de grado. Bogotá: Ingeniería Forestal, Facultad del Medio Ambiente y Recursos Naturales, Universidad Distrital Francisco José de Caldas; 85 pp.

Coronel EO. 1994. Fundamentos de las propiedades físicas y mecánicas de las maderas: Aspectos teóricos y prácticos para la determinación de las propiedades y sus aplicaciones. Santiago del Estero: Instituto de Tecnología de la Madera, Facultad de Ciencias Forestales.

Cunha MP, Pontes C, Cruz I, Cabral MF, Cunha Neto ZB, Barbosa APR. 1989. Estudio químico de 55 especies lenhosas para geração de energía em caldeiras. $3^{\circ}$ Encontro Brasilerio em Madeiras e em Estruturas de Madera. São Carlos, Brasil.

Dickson RL, Walker JCF. 1997. Selecting wood quality characteristics for pines. In: Zhang SY, Gosselin R, Chauret G (eds.). Timber Management Toward Wood Quality and End-Product Value. Proceedings of the
CTIA/IUFRO International Wood Quality Workshop, Quebec City. Part IV; 45-52 pp.

Gutiérrez A, Plaza F. 1967. Características fisico-mecánicas de las maderas españolas. Madrid: Institute Forestal de Investigaciones y Experiencias (IFIE), Ministerio de Agricultura; 103 pp.

Junta del Acuerdo de Cartagena. 1984. Manual de diseño para maderas del Grupo Andino. Lima, Perú.

Klinger W, Talero Y. 2001. Propuesta metodológica para la determinación de usos potenciales de la madera a partir de parámetros físico-mecánicos cuantitativos. Colomb Forestal. 7 (14): 7-24. URL disponible en: https://revistas.udistrital.edu.co/ojs/index.php/colfor/ article/view/3205/4693

Klinger W, Bohórquez A, Torres NF. 2002. Efectos de la aplicación de cinco tratamientos ignifugantes sobre la combustibilidad de las maderas de aceite maría, cedro macho, güino y sajo. Colomb Forestal. 7 (15): 29-40. URL disponible en: https://revistas.udistrital.edu.co/ ojs/index.php/colfor/article/view/3305/4805

Panshin AJ, De Zeeuw C. 1980. Textbook of wood technology: structure, identification, properties, and uses of the commercial woods of the United States and Canada. New York: McGraw-Hill Book Co.; 722 pp.

Schniewind AP. 1989. Concise encyclopedia of wood and Wood-based materials. Oxford: Pergamon Press.; 248 pp.

Teixeira do Vale A, Mourã MA, Lopes A. 2002. Quantificação e caracterização energética da madeira e casca de espécies do cerrado. Ciência Florestal, Santa Maria, 12 (1):71-80. URL disponible en: https://periodicos.ufsm. $\mathrm{br} /$ cienciaflorestal/article/view/1702/977

Torelli N. 1982. Estudio promocional de 43 especies forestales tropicales mexicanas. Programa de Cooperación Científica y Técnica México-Yugoslavia. Ciudad de México: Secretaría de Agricultura y Recursos Hidráulicos; 73 pp.

Williamson GB. 1975. Pattern and serial composition in an old growth beech-maple forest. Ecology. 56: 727-31.

WWF. (2013). Maderas de Colombia. Programa Subregional Amazonas Norte \& Chocó Darién. Bogotá: El Bando Creativo.

Zobel BJ, van Buijtenen JP. 1989. Wood variation. Its causes and control. Springer Series in Wood Science; 363 pp. 